infection is possible, but biological samples from a previous episode of vertigo were unavailable for testing.

Vestibular neuritis caused by enteroviral infection is reported in a 7-year-old male, and confirmed by isolation of enteroviral RNA in CSF and nasopharyngeal washings. (Ergul Y et al. Pediatr Neurol Jan 2006;34:45-46). Other etiologies listed include mumps, rubella, herpes simplex type 1, cytomegalovirus, and Epstein-Barr virus.

\title{
PROGNOSTIC VALUE OF CYTOCHROME C AND CYTOKINES IN ACUTE VIRAL ENCEPHALOPATHY
}

Serum cytochrome c and cytokines were evaluated as prognostic predictors in 29 children (ages 9 mos to 9 yrs 11 mos) with viral acute encephalopathies and multiple organ failure at Fukushima Medical University School of Medicine, Japan. Influenza A or AH3 was isolated in 13 patients, HHV-6 in 2, rotavirus (1), enterovirus (1), and the pathogen was unknown in 12. Serum samples collected in the initial phase showed elevated levels of cytochtome c (CTC-c), tumor necrosis factor-a (TNF-a), interleukin 6 (IL-6), soluble TNFreceptor 1 (sTNF-R1), and aspartate aminotransferase (AST), predictive of an unfavorable prognosis. A comparison of day 1 and day 2 measurements showed significant increases in patients who died compared to those who lived. CTC-c was the most sensitive of these markers (93\%), with $100 \%$ specificity, whereas TNF-a had $60 \%$ sensitivity and $100 \%$ specificity. IL-6 was $77 \%$ sensitive and $100 \%$ specific, and AST $82 \%$ and $83 \%$, respectively. These markers are proposed as guides to initial treatment and further intensive care. (Hosoya M, Kawasaki Y, Katayose M et al. Prognostic predictive values of serum cytochrome c, cytokines, and other laboratory measurements in acute encephalopathy with multiple organ failure. Arch Dis Child June 2006;91:469-472). (Respond: Dr M Hosoya, Dept of Pediatrics, Fukushima Medical University School of Medicine, Hikarigaoka-1, Fukushima 960-1295, Japan).

COMMENT. This study confirms previous reports of elevated serum concentrations of pro-inflammatory cytokines, cytokine receptors, and cytochrome $\mathrm{c}$ in children with acute influenza viral encephalopathy, prolonged convulsions, and multiple organ failure with high mortality $(30 \%)$. Brain edema develops on the day after neurologic onset, but fatal outcome is not predicted by neurological symptoms. The authors propose the measurement of cytochrome c and cytokines on day 1 and 2 of the illness as a predictor of prognosis and guide to early intensive treatment.

Surtees $\mathrm{R}$ and DeSousa C, in a perspective on this report (Influenza virus associated encephalopathy. Arch Dis Child June 2006;91:455-456), propose a sequence of events: influenza infection - hypercytokinemia - vascular inflammation - endothelial apoptosis cerebral edema - cerebral necrosis. One third die (multiorgan or brain stem failure), one third survive with sequelae and cerebral atrophy, and one third recover. Reduction of circulating cytokines by plasma exchange or anti-cytokine antibodies might limit vascular damage and prevent encephalopathy.

An elevated CSF/serum-albumin ratio, indicative of an impaired blood-brain barrier, was the earliest sign of acute viral encephalopathy, in a study of 22 patients, the majority having elevated interferon-a levels in CSF and serum (Clarke M et al. Dev Med Child Neurol 2006;48:294-300; Ped Neur Briefs April 2006;20:25-26). 